\title{
Seed-Mediated Growth of Gold Nanorods Using Silver Seeds: Effect of Silver Seeds Concentration and Growth Time
}

\author{
Suratun Nafisah ${ }^{1,2}$, Marlia Morsin ${ }^{1,2 *}$, Nur Anida Jumadi' ${ }^{2}$, Nafarizal Nayan ${ }^{1,2}$, Nur Zehan An'nisa Md Shah ${ }^{1,2}$, Nur \\ Liyana Razali ${ }^{1,2}$, Chin Fhong Soon ${ }^{1,2}$ \\ ${ }^{I}$ Microelectronics \& Nanotechnology - Shamsuddin Research Centre (MiNT-SRC), Institute of Integrated Engineering, Universiti Tun \\ Hussein Onn Malaysia, 86400 Parit Raja, Batu Pahat Johor, Malaysia \\ ${ }^{2}$ Department of Electronics, Faculty of Electrical and Electronic Engineering, Universiti Tun Hussein Onn Malaysia, 86400 Parit Raja, \\ Batu Pahat Johor, Malaysia \\ *Corresponding author E-mail: marlia@uthm.edu.my
}

\begin{abstract}
Seed-mediated growth method (SMGM) in preparation of gold nanoparticles becomes one of the most popular methods due to the simplicity of the experimental procedures and flexibility in structural modifications. In this paper, we report a new method for synthesizing gold nanoparticles using silver seeds. The effect of seed concentration and growth time are investigated in this work. By increasing the silver seed concentration, it is found that the color of the colloidal gold nanorods obtained are changed from light pink to reddish purple, the surface plasmon resonance band is shifted to the blue region whereas absorption spectra becomes narrower. The additional peak is also spotted when increasing silver seed concentration to $5 \mu$ l. Meanwhile, increasing the growth time from 5 to 240 minutes tends to increase the color concentration of the solution. Besides that, the absorbance of colloidal gold nanorods is also increased with an increase in the growth time whereas optimum growth time is found to be 45 minutes. FESEM characterization shows that gold nanoparticles shapes are dominated by nanorods with average length, width, and aspect ratio are $129.8 \mathrm{~nm}, 42.9 \mathrm{~nm}$, and 3.4, respectively. The energydispersive x-ray spectroscopy (EDX) shows the chemical composition of the synthesized sample is Gold (Au) with weight \% and atomic \% are 32.23 and 5.98, respectively. Besides that, signals from Carbon (C), Oxygen (O), and Indium (In) atoms were also recorded from EDS spectra. The present approach thus provides new method for synthesis gold nanoparticles with additional plasmon resonance peak thus it has very potential for application in plasmonic sensing.
\end{abstract}

Keywords: Gold Nanoparticles; Gold Nanorods; Localized Surface Plasmon Resonance; Plasmonic Sensor.

\section{Introduction}

Gold nanoparticles (GNPs) are most of the stable metal nanoparticles and one of the most promising materials for sensor [1], biosensor [2], catalyst [3] and drug delivery [4]. Recently, the fabrication of gold nanoparticles has attracted wide attention because of their unique optical properties. These optical properties are obtained from interaction of light and electron on the gold nanoparticles surface called localized surface plasmon resonance (LSPR) phenomenon [5]. The particular wavelength where this phenomenon occurs is strongly dependent on size, shape, and structure of gold nanoparticles [6].

Numerous studies have been carried out to GNPs with different shape such as spherical [7], rod [8], wire [9], plate [10], cages [11] and many other shapes. Gold nanorods (GNRs) exhibited two SPR modes i.e. transversal mode (t-SPR) and longitudinal mode (1SPR). These characteristics make GNRs very attractive to be used in many biological, sensing, and plasmonic applications. The consequence is researchers with a wide range of backgrounds extremely interested in studying GNRs production. There are three common methods used by researchers to produce GNRs, i.e. template method, electrochemical method, and seed-mediated growth method. In recent years, the seed-mediated growth method has been the most popular method to fabricate gold nanoparticles due to the simplicity of the experimental procedures and flexibility in structural modifications [12].

The synthesis of GNRs by seed-mediated growth method was first demonstrated in 2001 by Jana and co-workers [13]. They synthesize GNRs using 3 - $4 \mathrm{~nm}$ gold seeds prepared by reduction gold salt $\left(\mathrm{HAuCl}_{4}\right)$ with borohydride in the presence of sodium citrate. Meanwhile, the growth step was carried out by adding the seed solution to growth solution containing $\mathrm{HAuCl}_{4}, \mathrm{CTAB}$, ascorbic acid and silver. GNRs with various aspect ratios can be obtained by adding different volume of seeds to different samples of growth. In 2003, Nikhoobalt and El-Sayed developed a new variation of synthesizing GNRs by replacing sodium citrate with CTAB in the seed process. To synthesize GNRs with higher aspect ratio, they also introduced co-surfactant, namely BDAC, in the growth solution. Using this method, the aspect ratio of GNRs was controlled by adjusting the amount of silver ions in the growth solution and a yield as high as $99 \%$ for the GNRs was achieved [14] Recently, Murray and his co-researchers reported an improved SMGM for synthesizing GNRs by using aromatic additives with low CTAB concentration [15]. In their work, the seed solution was prepared following the procedures as reported by Nikhoobalt and El-Sayed. Growth solution was prepared by adding salicylatebased sodium salt for GNRs with 1-SPR less than $700 \mathrm{~nm}$ and salicylic acid for GNRs with 1-SPR greater than $700 \mathrm{~nm}$ in a solution containing gold salt, silver ion, ascorbic acid, and low concentration CTAB. As a result, the aspect ratio of GNRs could be 
controlled by adjusting the concentration of silver ions as well as the amount of seed solution used.

In this work, we introduced a new method to synthesize GNRs by replacing the seed nanoparticle with silver. Commonly, silver ion was known as a shape-directing agent in synthetic routine. When foreign metal ion, in this case is the silver ion, was introduced during seed process, the deposition during the growth process will be different with the regular process since the deposition mode on one metal to another metal surface can affected by their physicochemical properties. Therefore, the goals of this work are to investigate the effect of silver seeds concentration as well as the effect of growth time by means of SMGM.

\section{Experimental}

\subsection{Materials}

Hydrogen tetrachloroaurate $\left(\mathrm{HAuCl}_{4}\right)$, trisodium citrate, cetyltrimethylammonium bromide (CTAB), sodium borohydride $\left(\mathrm{NaBH}_{4}\right)$, and ascorbic acid were purchased from Sigma Aldrich (USA). Silver Nitrate $\left(\mathrm{AgNO}_{3}\right)$ was purchased from Emory (USA). All of the chemicals were used without further purification. DeIonized (DI) water with resistivity around $18.2 \mathrm{M} \Omega$, which was obtained from a Millipore RiOs water purification system was used for all solution preparations.

\subsection{Synthesis}

GNRs were synthesized using seed-mediated approach according to a procedure reported previously [16] with minor modifications. This process consists of two stages namely seeding and growth process. In the seeding process, silver seeds were prepared by reducing $\mathrm{AgNO}_{3}$ with $\mathrm{NaBH}_{4}$ in the presence of trisodium citrate. The $0.5 \mathrm{ml}$ of $0.01 \mathrm{M} \mathrm{AgNO}_{3}$ and $0.5 \mathrm{ml}$ of $0.01 \mathrm{M}$ trisodium citrate were added into $19 \mathrm{ml}$ DI in a glass vial, shaken for 30 seconds, and later, left undisturbed for 30 minutes at temperature $28^{\circ} \mathrm{C}$. Then, freshly prepared $0.5 \mathrm{ml}$ ice-cold $0.1 \mathrm{M} \mathrm{NaBH}_{4}$ was added into the solution. After adding $\mathrm{NaBH}_{4}$, the color of the solution changed to yellow, due to the formation of a silver nanoseeds The as-prepared silver seed was left undisturbed at temperature $28^{\circ} \mathrm{C}$ for 1 hour before further use. After that, the growth solution was prepared by adding $0.5 \mathrm{ml}$ of $0.01 \mathrm{M} \mathrm{HAuCl}_{4}$ and $0.12 \mathrm{ml}$ of $0.1 \mathrm{M}$ ascorbic acid into $20 \mathrm{ml}$ of $0.1 \mathrm{M}$ CTAB. After complete mixing, the solution color changed from yellow to colorless. Finally, growth process was carried out by added silver seeds solution into the growth solution. This mixture was left undisturbed at temperature $28^{\circ} \mathrm{C}$ before further use. Schematic of the sample preparation is shown in Figure 1.

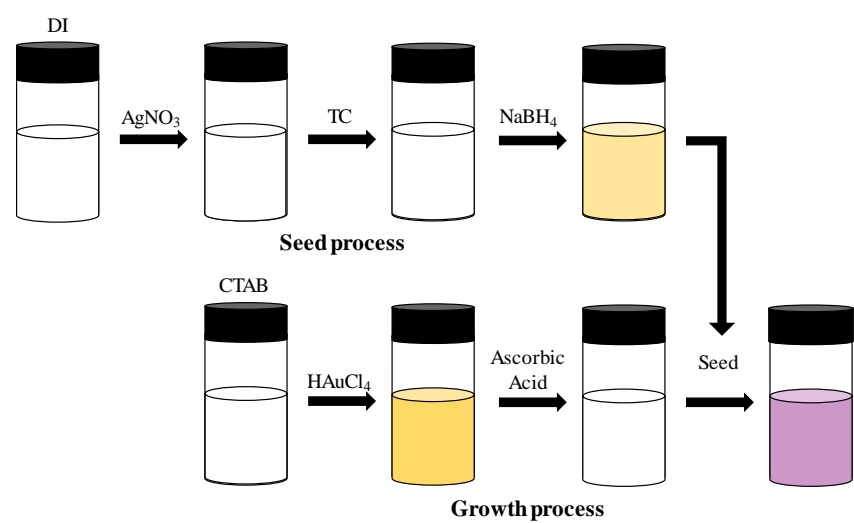

Fig. 1: The schematic of synthesizing gold nanorods using silver seeds.

In order to study the effect of seed concentration, seed solution was added into growth solution with three different concentrations, i.e. 1,5 , and $10 \mu l$. These samples were grown at growth time 1 hour and labeled as GNR1, GNR5 and GNR10. Meanwhile, to study the effect of growth time, the optimum seed solution concentration which is $5 \mu 1$ was grown at eight different growth time, i.e. 5, 10, 15, 30, 45, 60, 120 and 240 minutes. These samples were labeled as GNR5-5, GNR5-10, GNR5-15, GNR5-30, GNR545, GNR5-60, GNR5-120 and GNR5-240.

\subsection{Characterization}

UV-Vis absorption spectra were recorded by UV-1800 Shimadzu UV-Vis Spectrophotometer (Japan) at a wavelength ranging from 400 to $800 \mathrm{~nm}$. The surface morphology of the samples was obtained using a Joel JSM-7600F Schottky FESEM (USA) with an accelerating voltage at $5 \mathrm{kV}$. No metallization was required to observe the samples. The energy-dispersive x-ray spectroscopy (EDS) detector attached to the FESEM was used to analyze the elements of the sample. The voltage used for EDS also at $5 \mathrm{kV}$. For FESEM and EDS purpose, the sample was prepared by centrifuging $10 \mathrm{ml}$ of the growth solution at 4000 RPM using Sewage electric centrifugal machine model $800-1$ for 15 minutes. Then, the precipitate of the solution was dispersed in $10 \mathrm{ml}$ DI water and re-centrifuged for another 15 minutes to remove the excess surfactants. After second round of centrifugation, the precipitate was dispersed in $1 \mathrm{ml}$ of DI water to prepare a concentrated solution. $10 \mu \mathrm{l}$ of this solution was dropped on a clean ITO substrate $(5 \mathrm{~mm}$ x $5 \mathrm{~mm}$ ) and dried at $60{ }^{\circ} \mathrm{C}$.

\section{Results and Discussions}

The gold nanoparticles have been successfully synthesized using seed-mediated growth method. The schematic of reaction in seeding process is shown in Figure 2.

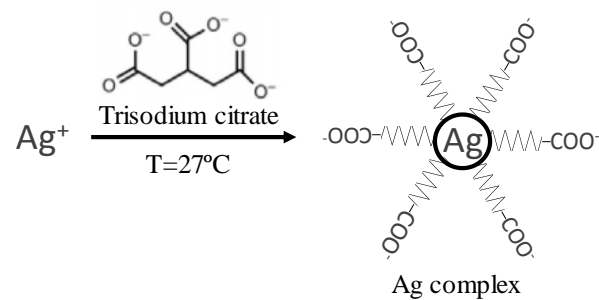

(a)

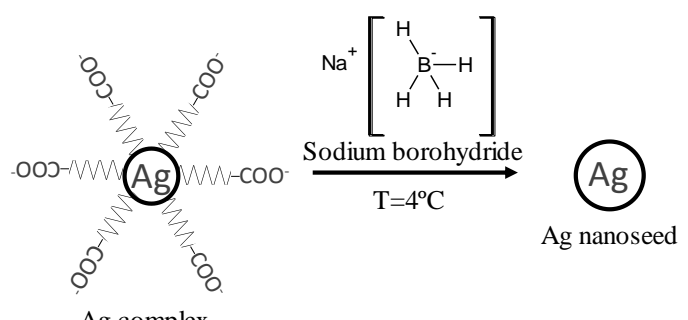

(b)

Fig. 2: The schematic of silver seeding process: (a) reduction process of $\mathrm{Ag}^{+}$ions to $\mathrm{Ag}$ complex in the presence of trisodium citrate and (b) reduction of $\mathrm{Ag}$ complex to $\mathrm{Ag}$ nanoseeds in the presence of sodium borohydride.

When $\mathrm{Ag}^{+}$ions and trisodium citrate were mixed, an $\mathrm{Ag}^{+}$ions capped with $\mathrm{COO}^{-}$head group and lead the formation of $\mathrm{Ag}$ complex [17] as seen in Figure 2a. As weak reducing agent (sodium borohydride) was added to the Ag complex, the solution turned to pale yellow (see Figure 1), indicates formation of silver seeds with size less than $5 \mathrm{~nm}$ as previously reported [18]. The reaction occurs in this condition is shown in Figure $2 b$.

Whereas, the reaction occurs in growth process is illustrated in Figure 3. When the ion $\mathrm{Au}^{3+}$ and CTAB surfactant were mixed, the mixture solution turned to orange (see Figure 1), indicating the 
formation of $\mathrm{AuBr}^{-}$[19]. Then, the solution turned to colorless when ascorbic acid was added (also see Figure 1). This indicates $\mathrm{Au}^{3+}$ ion was successfully changed to $\mathrm{Au}^{+}[20]$ as described in Figure 3a. This solution remains unchanged even after left for long time (more than 24 hours), indicates that $\mathrm{Au}^{3+}$ was not reduced to $\mathrm{Au}^{0}$ in the absence of seed solution. We assumed this is because of ascorbic acid is too weak to reduce $\mathrm{Au}^{3+}$ to $\mathrm{Au}^{0}$. When the silver nanoseeds solution was added, the growth solution slowly turned from colorless to pinkish which demonstrates that gold nanoparticles was formed as shown in Figure 1 and the reaction occurs as shown in Figure 3b. It is assumed that at first stage, silver nanoseeds act as catalyst in reduction of $\mathrm{Au}^{3+}$ to $\mathrm{Au}^{0}$ by ascorbic acid and then its surface play a role as nucleus in initial growth process.
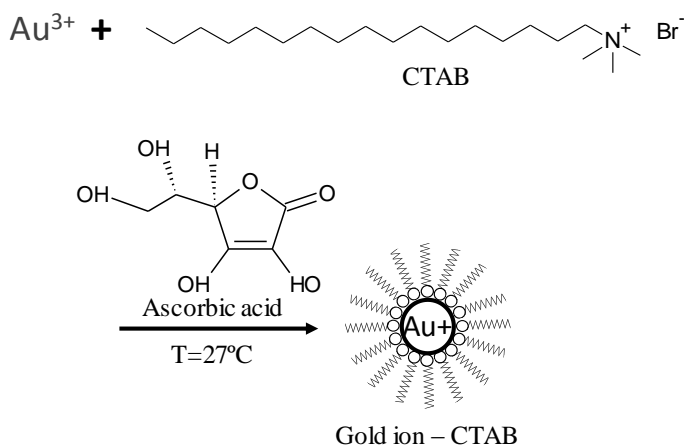

(growth solution)

(a)

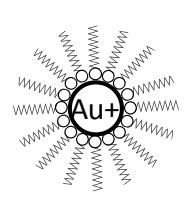

Growth solution

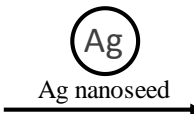

Fig. 3: The schematic of gold nanoparticles seeding process: (a) reduction process of $\mathrm{Au}^{3+}$ ions to $\mathrm{Au}^{+}$complex ions in the presence of ascorbic acid and (b) reduction of $\mathrm{Au}^{+}$complex to $\mathrm{Au}^{0}$ ( $\mathrm{Au}$ nanoparticles) assist by $\mathrm{Ag}$ nanoseeds.

\subsection{Effect of silver seed concentration}

Formation of gold nanoparticle in solution was confirmed by the appearance of color change in growth solution after a certain time of growth process. Photographs of aqueous gold nanoparticles synthesized with various silver seed concentration were shown in Figure 4.

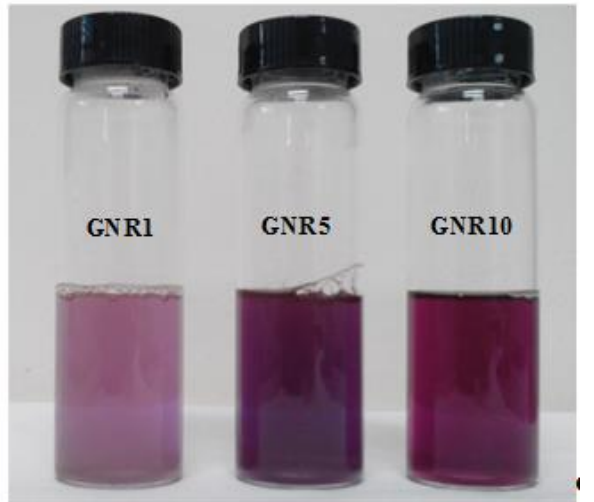

Fig. 4: Photograph of aqueous solutions of GNRs synthesized with various silver seed concentration.
When silver seed concentration $1 \mu \mathrm{l}$ added to growth solution, the color of colloidal gold nanoparticles is light pink. As the silver seed concentration was increased to $5 \mu$, colloidal gold nanoparticles in purple are produced. Continual increase of the silver seed concentration to $10 \mu \mathrm{l}$ produced reddish purple colloidal gold. This color difference indicates that GNPs produced have different sizes, shape or density [21].

UV-Vis absorption spectra of gold nanoparticles synthesized using various silver concentrations were shown in Figure 5. Increasing silver seed concentration from 1 to $10 \mu \mathrm{l}$ resulted in increasing in absorbance. This indicates the particle concentration obtained is increased [22] as a result of high metal nuclei. Increasing silver seed concentration also shifted SPR band to the blue region (SPR reading peak for GNR1, GNR5, and GNR10 are 562, 541, and 534 $\mathrm{nm}$, respectively) which correlated to a decrease in nanoparticles size [23]. Besides that, as the silver seed concentration increased, the absorption spectra also become narrower indicated that homogeneity of gold nanoparticles are increased [24]. Additional peak which indicates the shape changes also appeared by the increasing of silver seed concentration (GNR5). However, this additional peak became less prominent when silver seed concentration increased to $10 \mu \mathrm{l}$ (GNR10).

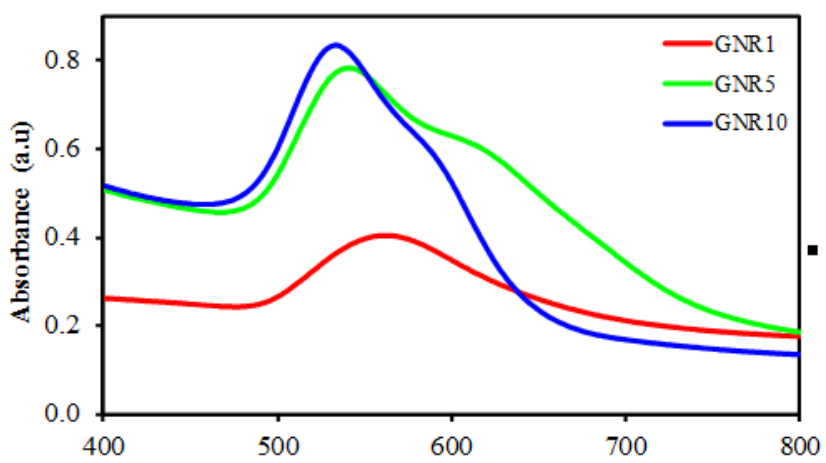

Fig. 5: UV-Vis absorption of GNRs synthesized with various silver seed concentration.

Based on the results obtained from UV-Vis absorption spectrum, a shape control mechanism of gold nanoparticles can be achieved by changing concentration of silver nanoseeds. The schematic illustration of gold nanoparticles shape control is shown in Figure 6. When the concentration of silver nanoseeds is $1 \mu 1$ (GNR1), the shapes of gold nanoparticles are in sphericals with only one SPR peak visible at $562 \mathrm{~nm}$. Two SPR peaks at $541 \mathrm{~nm}$ and $617 \mathrm{~nm}$ were observed in sample GNR5 indicating that gold nanoparticles obtained are in nonsphericals shape resembling nanorods characteristics. Two SPR wavelengths peaked at $534 \mathrm{~nm}$ and $590 \mathrm{~nm}$ could still be observed as the quantity of silver nanoseeds continuously increased. However, the second peak is blue shifted compared to the sample GNR5 (from $617 \mathrm{~nm}$ to $590 \mathrm{~nm}$ ), indicating that nanorods obtained have smaller aspect ratio (as well as its diameter and length). These results will be verified using FESEM.

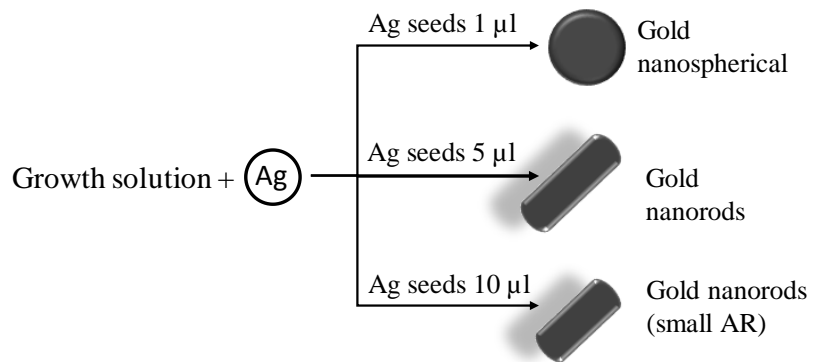

Fig. 6: Schematic illustration of the gold nanoparticles shape control by changing concentration of $\mathrm{Ag}$ nanoseeds. 


\subsection{Effect of growth time}

The color of colloidal gold nanoparticles synthesized using silver seed at concentration $5 \mathrm{ml}$ with different growth time were shown in Figure 7. It was observed that as the growth time extended, the color of the colloidal gold nanoparticles changes from light pink to dark purple. The first 5 minutes growth time changed the growth solution from colorless to light pink. When the growth time extended from 5 to 240 minutes, the color of colloidal gold nanoparticles slowly changing to dark purple.

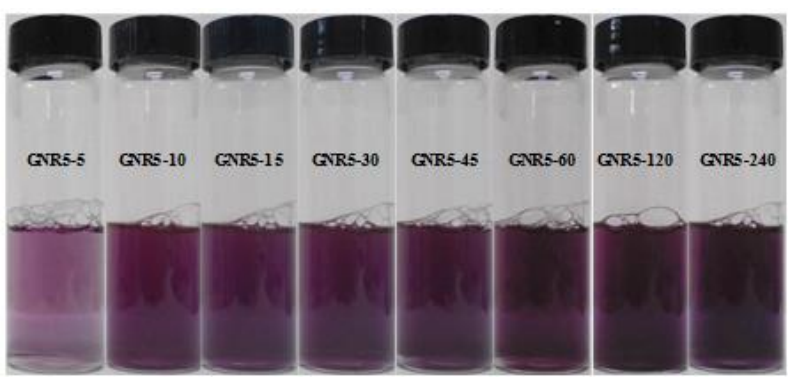

Fig. 7: Photograph of colloidal gold nanoparticles synthesized using silver seed with different growth time.

Absorption spectra of gold nanoparticles under similar condition was shown in Figure 8 and its reading of absorbance peaks was tabulated in Table 1. It showed that the absorbance increased with an increase in the growth time. Optimum absorbance obtained from a sample prepared at growth time of 45 minutes. When growth time extended over 45 minutes, the absorbance spectra were decreased. It was also revealed that sample GNR5-5 exhibited only one absorption peak, while other samples exhibited two absorption peaks. The first peak at wavelength $541 \mathrm{~nm}$ was associated with transversal surface plasmon resonance (t-SPR) of gold nanoparticles whereas the second peak associated with longitudinal surface plasmon resonance (1-SPR) was blue shifted from 640 to $625 \mathrm{~nm}$ as their growth time increased from 15 minutes to 240 minutes.

From the UV-Vis absorption spectra, it can be assumed that the growth of gold nanoparticles started with the formation of spherical shape as demonstrated in Table 1. However, as the addition of growth time, the growth of gold nanoparticles along the longitudinal direction faster than transversal direction, resulting nanorods shapes. Colloidal gold nanoparticles thermodynamically stable for growth time up to 30 minutes.

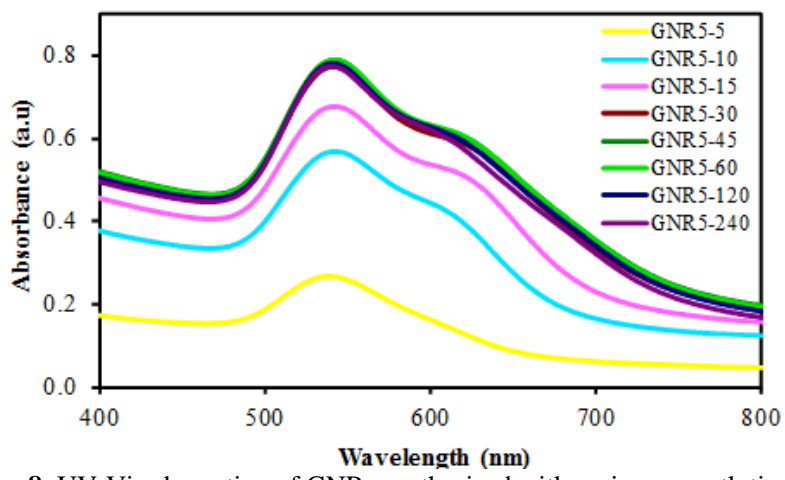

Fig. 8: UV-Vis absorption of GNRs synthesized with various growth time.

Table 1: UV-Vis absorbance result for the sample prepared at different growth time.

\begin{tabular}{|c|c|c|c|c|}
\hline \multirow{2}{*}{ Sample } & \multicolumn{2}{|c|}{ t-SPR } & \multicolumn{2}{c|}{ l-SPR } \\
\cline { 2 - 5 } & $\begin{array}{c}\text { Wavelength } \\
\text { (nm) }\end{array}$ & $\begin{array}{c}\text { Absorbance } \\
\text { (a.u) }\end{array}$ & $\begin{array}{c}\text { Wavelength } \\
\text { (nm) }\end{array}$ & $\begin{array}{c}\text { Absorbance } \\
\text { (a.u) }\end{array}$ \\
\hline GNR5-5 & 541 & 0.268 & - & - \\
\hline GNR5-10 & 541 & 0.569 & 634 & 0.342 \\
\hline GNR5-15 & 541 & 0.676 & 640 & 0.445 \\
\hline GNR5-30 & 541 & 0.772 & 640 & 0.534 \\
\hline
\end{tabular}

\begin{tabular}{|c|c|c|c|c|}
\hline GNR5-45 & 541 & 0.791 & 637 & 0.561 \\
\hline GNR5-60 & 541 & 0.790 & 636 & 0.563 \\
\hline GNR5-120 & 541 & 0.784 & 628 & 0.572 \\
\hline GNR5-240 & 541 & 0.772 & 625 & 0.557 \\
\hline
\end{tabular}

FESEM image of gold nanoparticles synthesized using optimum condition (silver seed concentration $5 \mathrm{ml}$ and growth time 45 minutes, GNR5-45) was shown in Figure 9. It was clearly seen from Figure 9a that the gold nanoparticles were produced, represented by white grain. However, the structure obtained was inhomogeneous. Higher FESEM magnification, i.e. 30K (Figure 9b) shows that the sample consists of several shapes such as rods, spherical, and irregular shape which highlighted by red, blue, and yellow circle, respectively. FESEM image with size 3.09 x 4.13 $\mu \mathrm{m}$ further analyzed using ImageJ to calculate the population of each shape. Figure 9d shows the detailed calculation of each structure in percentage. It is revealed that the majority of gold nanoparticles was nanorods $(50 \%)$ as shown in Figure $9 \mathrm{c}$, meanwhile the population of spherical and irregular shape were $29 \%$ and $21 \%$, respectively.

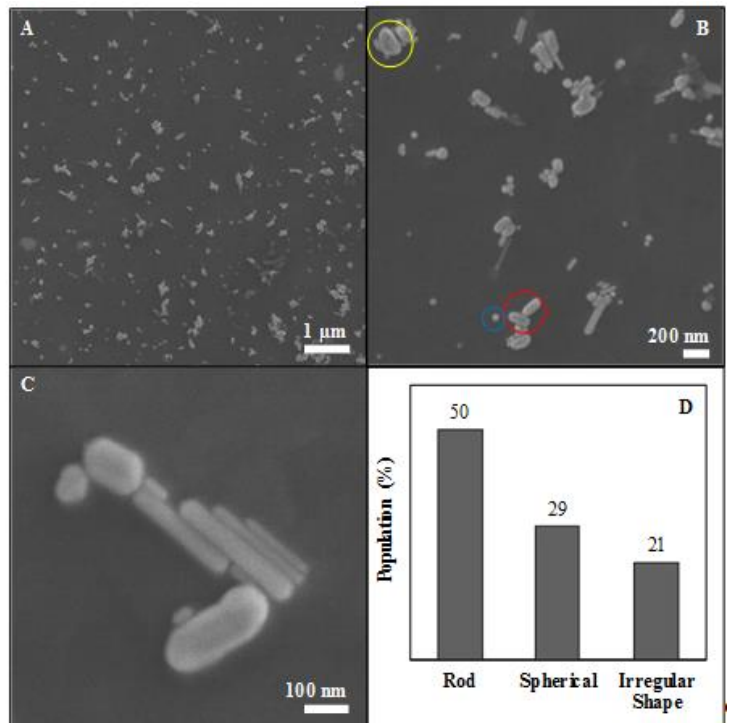

Fig. 9: FESEM image of gold nanoparticles produced following aforementioned protocol at optimum condition (GNR5-45) at magnification a) $10 \mathrm{~K}$, b) $30 \mathrm{~K}$, c) $100 \mathrm{~K}$ and d) graphical representations of the distribution of the gold nanoparticles shape.

Further, the size (length, width, and aspect ratio) distribution of gold nanorods were also calculated using Image J and the results were shown in Figure 10. Length of the gold nanorods was observed to be varied from 36 to $332 \mathrm{~nm}$ and width varies from 19 to $88 \mathrm{~nm}$. Minimum and maximum aspect ratios for the gold nanorods were 1.4 and 8.9 , respectively.

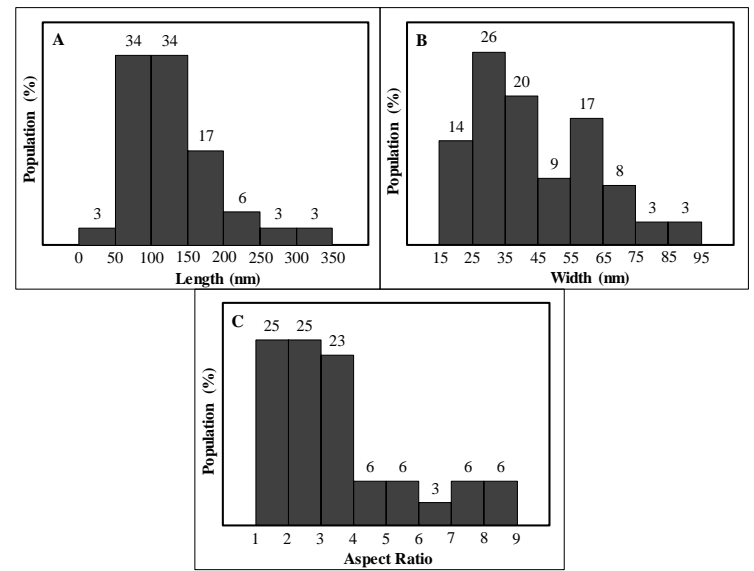

Fig. 10: Histogram of a) length, b) width and c) aspect ratio of gold nanorods from sample GNR5-45. 
To determine the chemical elements at the sample, an energy dispersive X-ray spectroscopy (EDS) analysis was conducted. The EDS spectrum of the sample was shown in Figure 11. The peaks of gold, carbon, oxygen, and indium were found in each LIS-GNP. The presence of gold peaks confirms that nanoparticles obtained are gold nanoparticles. Indium and oxygen peaks found in the EDS spectrum was contributed from the ITO substrate, since the ITO was made of $74 \% \mathrm{In}, 18 \% \mathrm{O}_{2}$, and $8 \% \mathrm{Sn}$. Meanwhile, the presence of carbon peak in EDS spectrum due to the interaction between the sample and the remaining environment in the FESEM chamber. It is also interesting to note that the silver peaks could not be observed from the spectrum. This finding suggests that the surface composition of the nanoparticles prepared using this method have phase purity of gold nanocrystals.

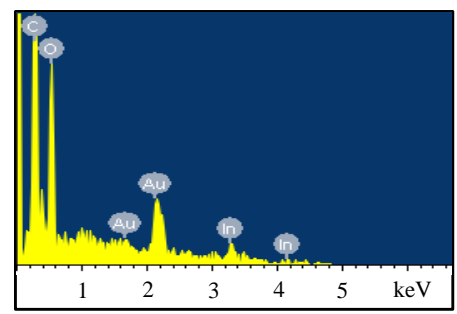

\begin{tabular}{|c|c|c|}
\hline Element & Weight \% & Atomic \% \\
\hline $\mathrm{C}$ & 17.34 & 51.20 \\
$\mathrm{O}$ & 14.44 & 32.01 \\
$\mathrm{In}$ & 34.99 & 10.81 \\
$\mathrm{Au}$ & 33.23 & 5.98 \\
\hline Total & 100 & 100 \\
\hline
\end{tabular}

Fig. 11: EDS spectrum of the sample GNR5-45.

\section{Conclusion}

A simple method for synthesis colloidal gold nanoparticles from silver seed has been demonstrated. In this study, the physical, optical and structural of gold nanorods were investigated. According to the results, it was found that silver seed concentration played a critical role in the formation of gold nanorods. It was found that silver seed concentration $5 \mu \mathrm{l}$ suitable for formation of gold nanorods. Below this concentration, gold nanospherical was obtained as indicated by only one SPR band. It was also found that increasing growth time lead to higher density of gold nanoparticles which was indicated by an increment of absorbance and reached optimum condition at growth time 45 minutes.

\section{Acknowledgment}

This work was supported by Universiti Tun Hussein Onn Malaysia under UTHM Contract Research Grant (U565-UTHM). The author would like to thank to Microelectronics \& Nanotechnology - Shamsuddin Research Centre (MiNT-SRC) for the laboratory facilities and Mr. Mohamad Syafiq Alias for the help performing the FESEM measurements. The authors also thank to Assoc. Prof. Mohd Zainizan Bin Sahdan for allowing using facilities at Semiconductor Nanomaterials Processing Research Laboratory.

\section{References}

[1] Priyadarshini E \& Pradhan N (2017), Gold nanoparticles as efficient sensors in colorimetric detection of toxic metal ions: a review, Sensor and Actuators B: Chemical, Vol. 238, pp. 888-902.

[2] Xinjun Y, Yang J \& Qinyuan C (2016), Applications of gold nanoparticles in biosensors, Nano LIFE, Vol. 6, No. 2, pp. 1-11.

[3] Leandro L, Camila PR, Aitor G, Jesum AF, Dario E \& Jairton D (2017), Revealing hydrogenation reaction pathways on naked gold nanoparticles, ACS Catalysis, Vol. 7, No. 4, pp. 2791-2799.

[4] Fen-Ying K, Jin-Wei Z, Rong-Fang L, Zong-Xia W, Wen-Juan W \&Wei W (2017), Unique roles of gold nanoparticles in drug delivery, targeting and imaging applications, Molecules, Vol. 22, No. 9 pp. $1445-1457$.

[5] Ryo T, Seiji Y, Kiichirou K, Kohei I \& Tatsuya T (2018), Gold ultrathin nanorods with controlled aspect ratio and surface modification: formation mechanism and localized surface plasmon resonance, Journal of the American Chemical Society, Vol. 140, No. 21 pp. 6640-6647.
[6] Shirin RK, Angus RG, Michael BC \& Andrew MD (2018), On the development of optical properties during thermal coarsening of gold nanoparticle composites, The Journal of Physical Chemistry C, Vol. 122, No. 22, pp. 12098-12105.

[7] NorShalihah MA, Farah NDI, Marlia M, Muhammad MS, Nur Anida J \& Marriatyi M (2017), Structural and optical properties of gold nanoshpericals in variation of growth time using seed mediated growth method, Journal of Telecomunication, Electronic and Computer Engneering, Vol. 9, No. 3-8, pp. 67-71.

[8] Sri N, Akrajas AU \& Muhamad MS (2012), The effect of nanoseed concentration on the aspect ratio of gold nanorod, Advanced Materials Research, Vol. 364, pp. 254-259.

[9] El Said AN, Edwin AB, Lise-Marie L, Febien D, Romuald P \& Guillaume V (2017), Surface-engineering of ultrathin gold nanowire: tailored self-assembly and enhanced stability, Langmuir, Vol. 33, No. 22, pp. 5456-5463.

[10] Marlia M, Muhamad MS, Akrajas AU \& Mohd ZS (2017), Gold nanoplates for a localized surface plasmon resonance-based boric acid sensor, Sensors, Vol. 17, No. 5, pp. 1-9.

[11] Sreejith R, Anindito S, Toru M \& Sakthi K (2017), Ultra-fast microwave aided synthesis of gold nanocages and structural maneuver studies, Nano Research, Vol. 10, No. 3, pp. 1078-1091.

[12] Jie C, Tong S \& Kenneth TVG (2014), Gold nanorod-based localized surface plasmon resonance biosensors: A review, Sensors and Actuators B: Chemical, Vol. 195, pp. 332-351.

[13] Nikhil RJ, Latha G \& Catherine JM (2001), Seed-mediated growth approach for shape-controlled synthesis of spheroidal and rod-like gold nanoparticles using a surfactant template, Advanced Materials, Vol. 13, No. 18, pp. 1389-1393.

[14] Babak N \& El-Sayed MA (2003), Preparation and growth mechanism of gold nanorods (NRs) using seed-mediated growth method, Chemistry of Materials, Vol. 15, No. 10, pp. 1957-1962.

[15] Xingchen Y, Linghua J, Humeyra C, Jun C, Guozhong X, Chen Z, Vicky DN, Yijin K, Nader E, Cherie RK \& Christopher BM (2012), Improved size-tunable synthesis of monodisperse gold nanorods through the use of aromatic additives, ACS Nano, Vol. 6, No. 3, pp 2804-2817.

[16] Suratun N, Marlia M, Nafarizal N, Nur Anida J, Nur Liyana R \& Nur Zehan ANMS (2017), Synthesis of gold nanorices on ITO substrate using silver seed-mediated growth method, International Journal of Integrated Engineering, Vol. 9, No. 4, pp. 1-5.

[17] Lei Q, Guangming Z, Cui L, Danlian H, Piao X, Chen Z, Ming C, Xigui L, Shiyu L, Bisheng L \& Huang Y (2018), Gold "rush" in modern science: fabrication strategies and typical advanced application of gold nanoparticles in sensing, Coordination Chemistry Reviews, Vol. 359, pp. 1-31.

[18] Simindokht R, Ali M \& Ali J (2017), Seed-mediated grown silver nanoparticles as a colorimetric sensor for detection of ascorbic acid, Spectrochimica Acta Part A: Molecular and Biomolecular Spectroscopy, Vol. 180, pp. 204-210.

[19] Changshun W, Caixia K, Jiejun Z, Xiaoling Z, Xiangfu W, Hongchen L \& Daning S (2010), Synthesis of high-yield gold nanoplates: fast growth assistant with binary surfactants, Journal of Nanomaterials, Vol. 2010, pp. 1-9.

[20] Jianhua S, Mingyun G, Tongming S, Cuiling G, Zheng X \& Jianmin Z (2008), Selective synthesis of gold cuboid and decahedral nanoparticles regulated and controlled by $\mathrm{Cu}^{2+}$ Ions, Crystal Growth \& Design, Vol. 8, No. 3, pp. 906-910.

[21] Luty-Blocho M, Wojnicki M, Grzonka J, Kurzydlowski KJ \& Fitzner K (2018), Linking the gold nanoparticles formation kinetics with their morphology, International Journal of Chemical Kinetics, Vol. 50, No. 3, pp. 204-214.

[22] Young JK, Lewinski NA, Langsner RJ, Kennedy LC, Satyanarayan A, Nammalvar V, Lin AY \& Drezek RA (2011), Size-controlled synthesis of monodispersed gold nanoparticles via carbon monoxide gas reduction, Nanoscale Research. Letters, Vol. 6, No. 1, p. 428.

[23] Debashish A, Bidhan M, Sanjib D \& Asoke KS (2017), Theoretical prediction of absorbance spectra considering the particle size distribution using mie theory and their comparison with the experimental UV-Vis spectra of synthesized nanopariticles, Spectroscopy Letters, Vol. 51, No. 3, pp. 1-5

[24] Agampodi SDSI, Sean FJ, Ren AO \& Tuan VD (2018), Manipulation of the geometry and modulation of the optical response of surfactant-free gold nanostars: a systematic bottom-up synthesis, ACS Omega, Vol. 3, No. 2, pp. 2202-2210. 